OPEN ACCESS

Edited by: Xiawei Cheng,

East China University of Science and

Technology, China

Reviewed by:

Abhik Saha,

Presidency University, India

Wei Zhao,

City University of Hong Kong, Hong

Kong SAR, China

*Correspondence:

Peng Cheng

pengchengchengdu@126.com

Specialty section:

This article was submitted to

Experimental Pharmacology and Drug

Discovery,

a section of the journal

Frontiers in Pharmacology

Received: 29 November 2021

Accepted: 30 December 2021

Published: 25 January 2022

Citation:

Jin Z, Chenghao Y and Cheng P (2022)

Anticancer Effect of Tanshinones on

Female Breast Cancer and

Gynecological Cancer.

Front. Pharmacol. 12:824531.

doi: 10.3389/fphar.2021.824531

\section{Anticancer Effect of Tanshinones on Female Breast Cancer and Gynecological Cancer}

\author{
Zhou Jin $^{1,2}$, Yu Chenghao ${ }^{2}$ and Peng Cheng ${ }^{1,2,3 *}$ \\ ${ }^{1}$ State Key Laboratory of Southwestern Chinese Medicine Resources, Chengdu University of Traditional Chinese Medicine, \\ Chengdu, China, ${ }^{2}$ Basic Medical College, Chengdu University of Traditional Chinese Medicine, Chengdu, China, ${ }^{3}$ College of \\ Pharmacy, Chengdu University of Traditional Chinese Medicine, Chengdu, China
}

Female breast cancer, ovarian cancer, cervical cancer, and endometrial cancer are the most common tumors and the most common causes of cancer-related mortality worldwide in women. Drugs derived from natural plants play important roles in malignant tumor therapy. Salvia miltiorrhiza is a commonly used Chinese herb which has been used in the treatment of liver diseases and cardiovascular diseases because of its positive effect of promoting blood circulation, increasing oxidative stress, and removing blood stasis. Recently, studies have found that fat-soluble components of Salvia miltiorrhiza such as tanshinone II, tanshinone I, cryptotanshinone, and dihydrotanshinone I displayed good antitumor activity in vivo and in vitro for gynecological cancer by different molecular mechanisms. In this study, the latest research progress on the antitumor effect and mechanism of tanshinone compounds in breast cancer and gynecological cancer was reviewed to provide references for the research and clinical application of these compounds (tanshinone II, tanshinone I, cryptotanshinone, and dihydrotanshinone I).

\footnotetext{
Keywords: gynecological cancer, tanshinones, molecular mechanism, traditional Chinese medicine, female breast cancer
}

\section{INTRODUCTION}

Female breast cancer, ovarian cancer, cervical cancer, and endometrial cancer are the most common tumors and the most common causes of cancer-related mortality worldwide in women. Surgical resection, radiotherapy, and chemotherapy remain effective curative treatments for early-stage gynecological tumors (DeSantis et al., 2014; Yadav et al., 2017; Amant et al., 2019; Coughlin, 2019). Unfortunately, only a minority of patients are candidates for surgical resection. Systemic chemotherapy has been widely employed in clinical treatment of female breast cancer and gynecological tumors, which increases the median survival time of patients with advanced gynecological tumors (Ponde et al., 2019; Burstein, 2020; Tsuyoshi et al., 2020). The commonly used chemotherapy drugs include natural anticancer agents from herb, alkylating agents, antimetabolites (methotrexate and cyclophosphamide), antitumor antibiotics, and platinum analogs (Gu et al., 2016; Kim et al., 2020; Li et al., 2020; Liu et al., 2020). Drugs derived from natural plants play important roles in malignant tumor chemotherapy. Therefore, screening new anticancer plant medicine has become one of the hotspots for tumor therapy in recent years.

Traditional Chinese medicine (TCM) possesses a long history and a lot of successful experiences. Lots of traditional Chinese medicines such as Radix Kansui, Rheum rhabarbarum, and Cinobufotalin 


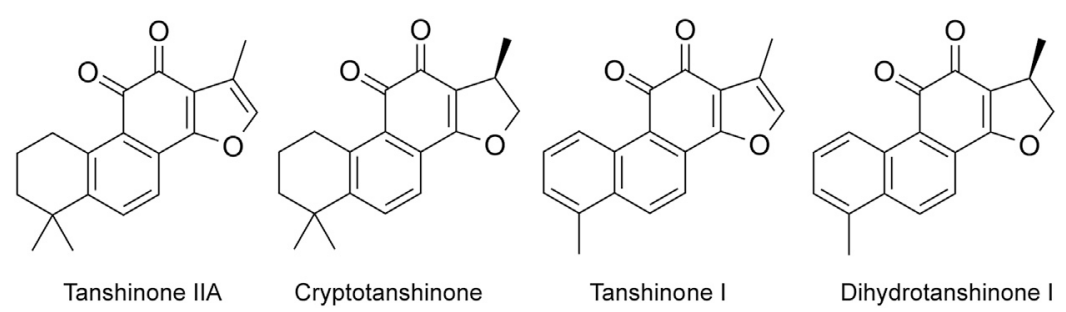

FIGURE 1 | Main fat-soluble components in Salvia miltiorrhiza (Tanshinone IIA, cryptotanshinone, tanshinone I, and dihydrotanshinone I).

can be used to inhibit breast cancer in vitro and in vivo by regulating differentially expressed genes and signaling pathways (Li J. et al., 2019; Liu YT. et al., 2019; Yang Z. et al., 2021). Many compounds or monomers from TCM such as oridonin, berberine, and matrine have displayed some antitumor effects in vitro and in vivo by inhibiting the proliferation of various cancer cells.

Salvia miltiorrhiza is a commonly used Chinese herb. It is believed in TCM that Salvia miltiorrhiza is good at promoting blood circulation and removing blood stasis. Li Shizhen said that Salvia miltiorrhiza has an effect in Siwu decoction in the Compendium of Materia Medica. Salvia miltiorrhiza is mainly produced in Sichuan, Shanxi, Hebei, Jiangsu, and Anhui, and its medicinal parts are roots and rhizomes. In the National Pharmacopoeia (2020 Edition), the efficacy of Salvia miltiorrhiza is summarized as removing blood stasis and alleviating pain, promoting blood circulation and dredging meridians, and clearing heart and eliminating annoyance. It is used for irregular menstruation, amenorrhea, accumulation of symptoms, chest and abdomen tingling, heat arthralgia pain, sore swelling, restlessness, hepatosplenomegaly, and angina pectoris. Salvia miltiorrhiza has been used in the treatment of liver diseases and cardiovascular diseases because of its positive effect of promoting blood circulation and removing blood stasis.

The effective components of Salvia miltiorrhiza include fatsoluble and water-soluble components. Fat-soluble components include tanshinone I, dihydrotanshinone I, tanshinone IIA, and tanshinone IIB (Figure 1). Water-soluble (phenolic acids) components include Danshensu, salvianolic acid A, salvianolic acid B, purple oxalic acid, protocatechuic aldehyde, and rosmarinic acid. Modern pharmacological studies have shown that these bioactive components have anti-inflammatory, antioxidative stress, antitumor and anti-myocardial ischemia effect, and can inhibit left ventricular hypertrophy, dilate blood vessels, resist atherosclerosis, protect brain tissue, resist thrombosis, improve microcirculation, promote fracture healing, significantly inhibit pulmonary fibrosis, and activate immunity.

In recent years, it has been found that fat-soluble components, namely, tanshinones, have good antitumor activity in vivo and in vitro. In this study, the latest research progress on the antitumor effect and mechanism of tanshinone compounds in breast cancer and gynecological cancer was reviewed to provide references for the research and clinical application of these compounds.

\section{TANSHINONES INHIBIT CELL PROLIFERATION}

In breast cancer, tanshinone IIA significantly inhibits colony formation and BrdU incorporation by a dose- and timedependent inhibitory effect on MCF-7 cells (IC50 $=0.25 \mathrm{mg} / \mathrm{ml}$ ), and tumor growth in negative human breast IDC-xenografted animal model, which is attributed to apoptosis induced through upregulation and downregulation of multiple genes involved in cell proliferation and apoptosis (Wang et al., 2005; Fu et al., 2014). Other studies have shown that tanshinone IIA significantly downregulated the viability of ER-positive human breast cancer cells (MCF-7) and ER-negative human breast cancer cells (MDA-MB231) by dose- and time-dependent manners in vitro through downregulating the expression of P53 and Bcl-2 ( $\mathrm{Lu}$ et al., 2009). According to another study, tanshinone IIA inhibits the growth of three kinds of breast cancer cells, such as MDA-MB453, SKBR3, and MDAMB231 cell lines (Gong et al., 2012). Su et al. found that tanshinone IIA suppresses tumor growth in MDA-MB-231 xenografted animal model by decreasing Erb-B2 and NF- $\mathrm{Bp} 65$ expression, and increasing caspase-3 expression and $\mathrm{Bax} / \mathrm{Bcl}-\mathrm{xL}$ ratio ( $\mathrm{Su}$ and $\mathrm{Lin}$, 2008; Su et al., 2012). Yan et al. showed that tanshinone IIA blocks cell proliferation and increased cell apoptosis on human breast cancer BT-20 cells by activating ER stress and MAPK pathway (increasing caspase-12, GADD153, and phospho-p38 protein expression and downregulating Bcl-xL and phospho-ERK expression) (Yan et al., 2012). Nicolin et al. also found that tanshinone IIA inhibits the proliferation of MCF-7 and MD-MB231 cells by inducing cell apoptosis and blocking cell cycle (Nicolin et al., 2014). Tanshinone I is one of the vital fat-soluble monomer components from the traditional herbal medicine, Salvia miltiorrhiza Bunge (Wang et al., 2019; Zhou et al., 2020a). It has been found that Tanshinone I has a cytotoxicity effect on breast cancer cells in a dose-dependent manner. The molecular mechanisms demonstrated that tanshinone I induces G0/G1 phase arrest in MCF-7 cells and both S and G2/M phase arrests in MDA-MB-231 cells by downregulating cell cycle markers such as cyclin D, CDK4, and cyclin B protein levels, and promoted cell apoptosis by increasing the protein level of pro-apoptosis-related proteins [cleaved PARP (c-PARP)] and reducing antiapoptosis-related protein (Bcl-2) (Gong et al., 2012). Tanshinone I inhibits the proliferation of MDA-MB-231 cells and MCF-7 cells, and induces cell apoptosis by decreasing the Bcl-2/Bax ratio and activating cleavage of caspase- 3 in cancer cells (Nizamutdinova et al., 


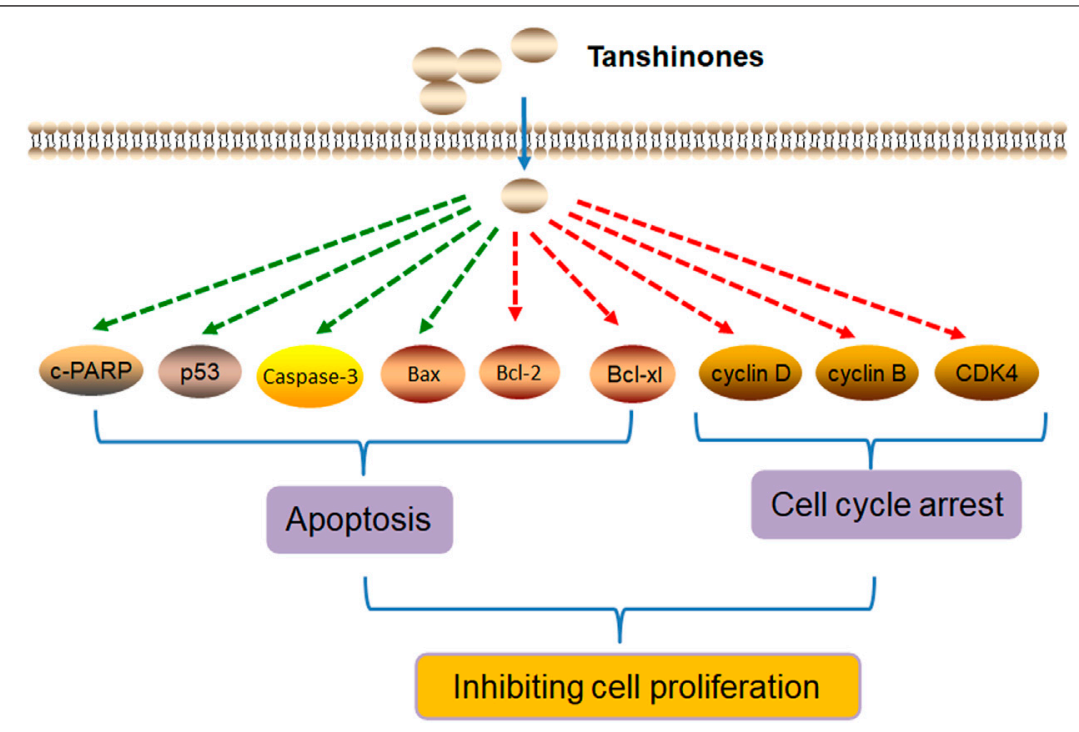

FIGURE 2 | Molecular mechanism of tanshinones inhibiting cell proliferation. Tanshinones inhibit cancer cell proliferation by inducing cell apoptosis and cell cycle arrest. c-PARP, pro-apoptosis-related proteins (cleaved PARP; caspase-3, cysteine aspastic acid-specific protease 3; Bax, Bcl2-associated X protein; Bcl-2, B-cell lymphoma-2; Bcl-xl, Bcl2-associated XI protein; CDK4, cyclin-dependent kinase 4).

2008b). Tanshinone IA also weakens cell proliferation by inhibiting DNA synthesis, inducing apoptosis, and arresting cell cycle of ERnegative human breast cancer cells (Jing et al., 2007). In summary, tanshinones can inhibit the growth of breast cancer cells by inducing cell apoptosis and cell cycle arrest in vitro and in vivo. At the molecular level, tanshinones can promote pro-apoptotic protein expression, and reduce anti-apoptotic protein expression and cell cycle-related protein expression.

In ovarian cancer, tanshinone IIA significantly inhibits cell growth and aggressiveness by inducing cell apoptosis, which is mediated by the $\mathrm{PI} 3 \mathrm{~K} / \mathrm{AKT} / \mathrm{JNK}$ signaling pathway in ovarian cancer (Zhang et al., 2019). Other studies showed that tanshinone IIA has a growth inhibitory effect by inducing cell apoptosis and downregulating survivin expression in ovarian cancer cells (Jiao and Wen, 2011; Li N. et al., 2018). Our studies showed that tanshinone IIA inhibits the growth of ovarian cancer cells in a dose-dependent manner by inducing G2/M phase arrest and cell apoptosis (Zhou et al., 2020c). In ovarian cancer, tanshinone IA significantly suppresses cell proliferation in vitro and tumor growth in vivo by inducing apoptosis and promoting autophagy via the inactivation of the PI3K/ AKT/mTOR pathway in ovarian cancer (Zhou et al., 2020a). Another bioactive component dihydrotanshinone I significantly inhibits the proliferation of ovarian cancer cells in vitro and in vivo by modulating the PI3K/AKT signaling pathway (Wang et al., 2020). Cryptotanshinone inhibits cellular glycolysis-induced cell growth and proliferation by suppressing the STAT3/SIRT3/HIF-1a signaling pathway (Yang et al., 2018).

In cervical cancer, tanshinone IIA inhibits cell viability, resulting in a $72.7 \%$ reduction in tumor volume in vivo by inducing apoptosis and decreasing glycolysis (Liu Z. et al., 2019). It is reported in other studies that tanshinone IIA inhibits the proliferation of human cervical cancer CaSki, SiHa, HeLa, and C33a cells in vitro, leading to a $66 \%$ reduction in the volume of cervical cancer xenograft in vivo by inducing $\mathrm{S}$ phase cell cycle arrest and p53-mediated apoptosis (Munagala et al., 2015). Tanshinone IIA inhibits the proliferation of HeLa cells by inducing pyroptosis via upregulating the expression of GSDMD and miR-145 (Tong et al., 2020). Two proteomic analyses revealed that tanshinone IIA possesses strong growth inhibition effect against human cervical cancer cells in dose- and time-dependent manners by regulating the expressions of proteins involved in microtubule assembly, leading to $\mathrm{G}$ (2)/M phase arrest, mitochondria-mediated intrinsic apoptosis, and endoplasmic reticulum stress (Pan et al., 2010; Pan et al., 2013). Tanshinone I suppresses the proliferation by inhibiting ELK1 and downregulating KRAS-AKT axis in cervical cancer cells (Dun and Gao, 2019). Tanshinone IIA and cryptotanshinone have cytotoxic and apoptotic effects against HeLa cell lines (Zaker et al., 2017). Cryptotanshinone inhibits the proliferation by inducing G0/G1 phase arrest and apoptosis of cervical cancer HeLa cells (Ye et al., 2010). Dihydrotanshinone I displays an antiproliferative effect on human cervical cancer cells (Luo et al., 2018).

In endometrial cancer, tanshinone I blocks the proliferation of the human endometrial carcinoma HEC-1-A cells in a dose-dependent manner by causing apoptosis and increasing the ROS level ( $\mathrm{Li}$ Q. et al., 2018). In summary, tanshinones can significantly suppress the proliferation of gynecological cancer cells in vitro and in vivo by inducing cell cycle arrest and apoptosis.

In summary, tanshinones inhibit cell proliferation by inducing cell apoptosis and cell cycle arrest (Figure 2).

\section{TANSHINONES BLOCK METASTASIS PHENOTYPE}

Invasion and metastasis are one of the main malignant phenotypes of breast cancer cells. It has been found that tanshinone IIA as an active 


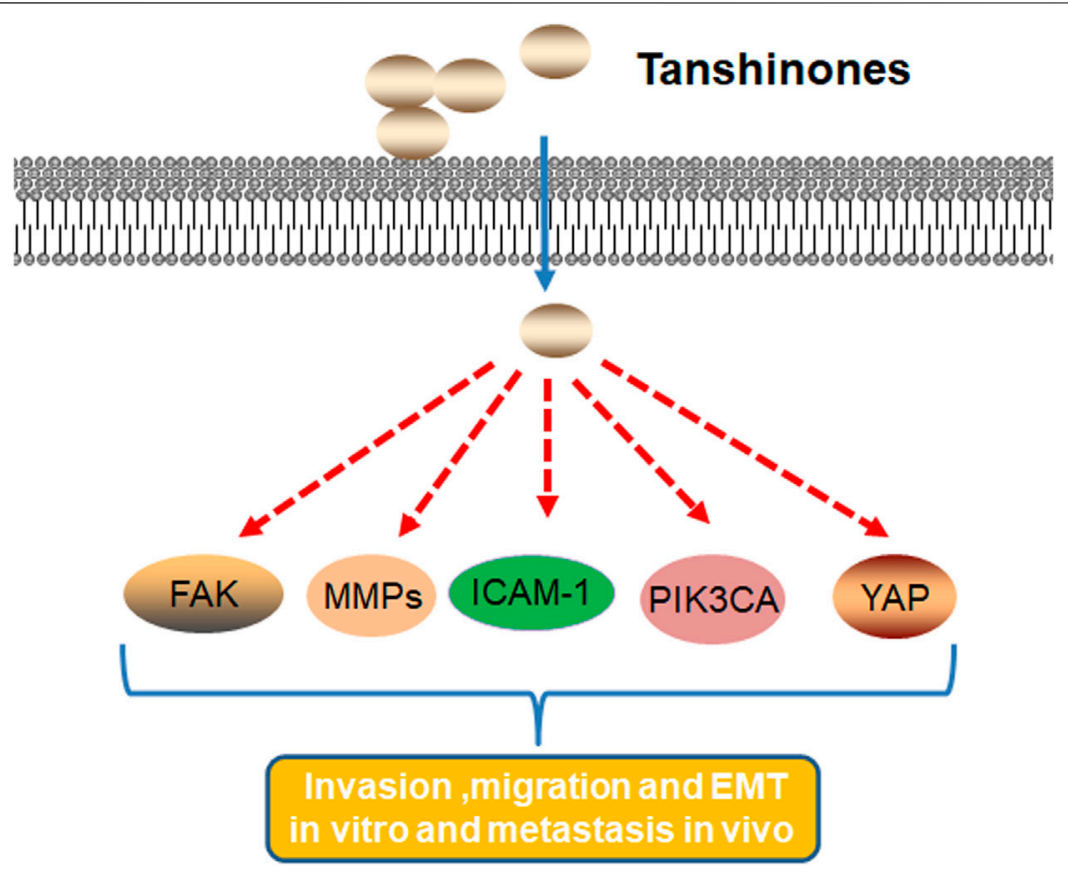

FIGURE 3 | Molecular mechanism of tanshinones inhibiting cell migration, invasion, metastasis, and EMT. Tanshinones inhibit protein expression in migration, invasion, metastasis, and EMT. FAK, focal adhesion kinase; MMPs, matrix metalloproteinases; ICAM-1, intercellular cell adhesion molecule-1; PIK3CA, phosphatidylinositol-4,5-bisphosphate 3-kinase catalytic subunit alpha; YAP, Yes1 associated transcriptional regulator.

constituent of Salvia miltiorrhiza Bunge ameliorates hypoxia-induced epithelial-mesenchymal transition (EMT) in breast cancer MCF-7 cell lines (Fu et al., 2014). Recent studies have shown that tanshinone I significantly downregulates adhesion of MDA-MB-231 cells to human umbilical vein endothelial cells (HUVECs) by reducing ICAM-1 and VCAM-1 expressions in HUVECs and inhibiting the migration of MDA-MB-231 cells through extracellular matrix (Nizamutdinova et al., 2008a). Additionally, tanshinone I inhibits cancer metastasis in the MDA-MB-231 xenografted animal model (Nizamutdinova et al., 2008a). Except nature products, some analogs of tanshinones also exhibit antitumor effects. For example, tanshinone-IIA-based analogs of imidazole alkaloid can effectively inhibit the migration and invasion of MDA-MB-231 cells and metastasis of MDA-MB-231 cells in zebrafish xenografts (Wu et al., 2018). Other studies showed that 2phenyl-1H-imidazole-based tanshinone IIA derivatives inhibit the invasion and migration of breast cancer cells and metastasis of MDA-MB-231 cells in xenografts. In summary, tanshinone and its derivatives can inhibit invasion, migration, and EMT in vitro and metastasis of breast cancer cells in vivo.

In ovarian cancer, Zhou et al. found that tanshinone IIA inhibits the migration of A2780 and ID-8 ovarian cancer cells by inhibiting focal adhesion kinase phosphorylation (Zhou et al., 2020c). Another bioactive component, namely, cryptotanshinone, can suppress migration and invasion of ovarian cancer A2780 cells by dramatically inhibiting MMP-2 and MMP-9 expression (Jiang et al., 2017). Wang et al. reported that dihydrotanshinone I can suppress the migration and invasion of ovarian cancer cells in a concentration-dependent manner by downregulating phosphatidylinositol-4,5-bisphosphate 3-kinase catalytic subunit alpha (PIK3CA) (Wang et al., 2020).
In cervical cancer, tanshinone IIA inhibits cervical cancer stem-like cells' migration and invasion in dose- and timedependent manners by reducing YAP mRNAs' stability and transcriptional activity (Qin et al., 2018).

In summary, tanshinones block migration, invasion, metastasis, and EMT by downregulating gene expression such as FAK, MMPs, ICAM-1, PIK3CA, YAP, and Yes1 (Figure 3).

\section{TANSHINONES ENHANCE CHEMOSENSITIVITY AND RADIOSENSITIVITY}

Chemotherapy is widely employed in clinical treatment of breast cancer, such as cisplatin, paclitaxel, mitoxantrone, gemcitabine antimetabolites, antitumor antibiotics, platinum analogs, and natural anticancer agents. The development of chemoresistance is the main reason for treatment failure. Recent studies have shown that nature agents and Chinese medicine play a critical role in improving the sensitivity of cancer cells to chemotherapeutic drugs. Tanshinone IIA ameliorates hypoxia-induced doxorubicin (Dox) resistance on breast cancer MCF-7 cells (Fu et al., 2014). Tanshinone IIA promotes the chemosensitivity of breast cancer MCF-7 cells to Dox in a dosedependent manner by downregulating the expression of efflux $\mathrm{ABC}$ transporters including P-gp, BCRP, and MRP1 (Li and Lai, 2017). Tanshinone IIA increases chemosensitivity of ER-positive breast cancer MCF-7 cells to chemotherapy agent Taxol by inhibiting Tau expression (Lin et al., 2018). Tanshinone IIA can also promote sensitivity of breast cancer cells to Dox by suppressing the PTEN/AKT pathway and the expression of efflux $A B C$ 


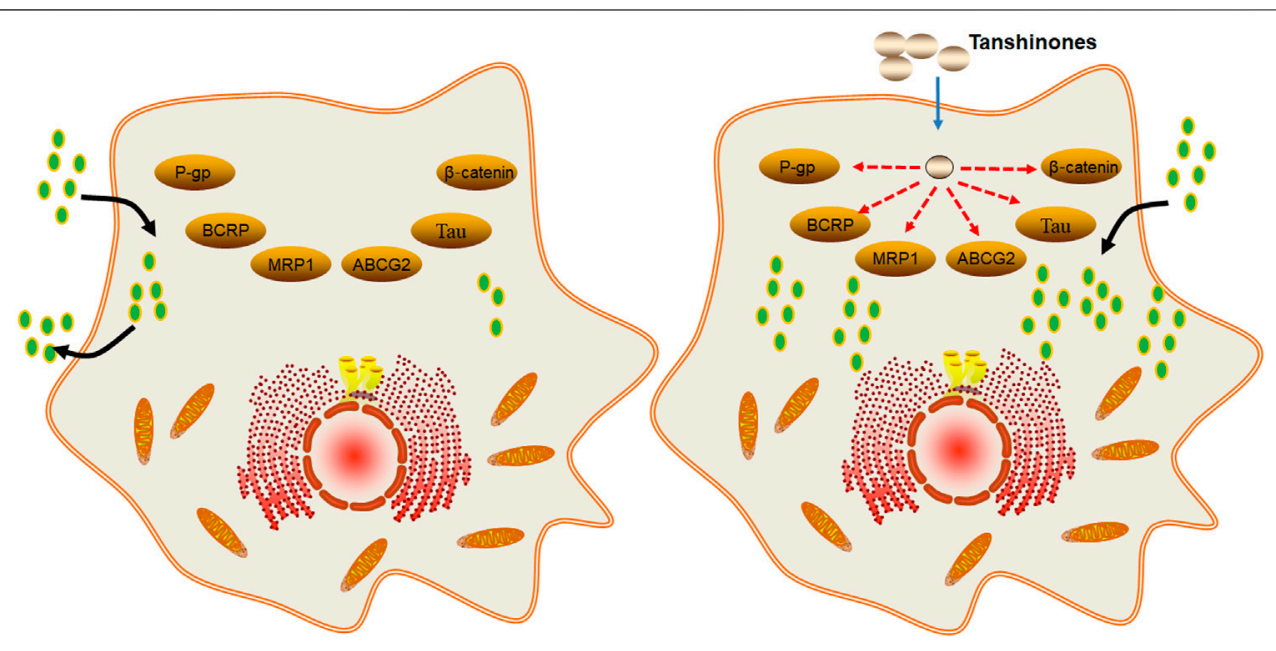

FIGURE 4 | Molecular mechanism of tanshinones increasing the efficacy of chemotherapy and radiotherapy. Tanshinones inhibit protein expression of P-gp, BCRP, MRP1, ABCG2, Tau, and $\beta$-catenin. P-gp, P-glycoprotein; multidrug resistance-associated protein 1; ABCG2, ATP binding cassette subfamily G member 2; Tau, microtubule-associated protein; $\beta$-catenin, beta-cadherin-associated protein.

transporters including P-gp, BCRP, and MRP1 (Li K. et al., 2019). Tanshinone IIA promotes the chemosensitivity of breast cancer MCF-7 cells to Dox by inhibiting $\beta$-catenin nuclear translocation (Li et al., 2021). Recent studies have shown that tanshinone IA exhibits MDR reversing potential to human ER-negative breast cancer cells by downregulating BCRP/ABCG2 expression in cancer cells (Jing et al., 2007). Therefore, tanshinone, as an effective chemosensitizer, is used in combination with chemotherapeutic drugs in the treatment of breast cancer.

Tanshinone IIA promotes TRAIL sensitization by upregulating DR5 through the ROS-JNK-CHOP signaling axis in human ovarian carcinoma cell lines (Chang et al., 2015). Our study showed that natural compound tanshinone I enhances the efficacy of paclitaxel chemotherapy on ovarian cancer (Zhou et al., 2020b). Other studies confirmed that cryptotanshinone can sensitize ovarian cancer cells to chemotherapy drug cisplatin (Jiang et al., 2017). In summary, tanshinones facilitate chemotherapy susceptibility in ovarian carcinoma cell to supply the potential clinical use of ovarian carcinoma cells.

Dihydrotanshinone I as the radiosensitizer significantly increases the efficacy of irradiation on HeLa cells by upregulation of p21 expression and caspase- 3 activity and downregulation of cyclin B1, p34 (cdc2) expression, which has potential in the treatment of human cervical cancer (Luo et al., 2018).

In summary, tanshinones can enhance the chemosensitivity and radiosensitivity by reducing the expression of efflux $\mathrm{ABC}$ transporters such as P-gp, BCRP, and MRP1 (Figure 4).

\section{TANSHINONES INHIBIT ANGIOGENESIS}

Angiogenesis plays a key role in tumor proliferation and metastasis and contributes to the cancer progression (Folkman, 2002; Yousefi et al., 2021). To provide enough oxygen and nutrients to tumor growth, tumor vessels are regulated by multiple angiogenic growth factors such as vascular endothelial growth factor (VEGF), basic fibroblast growth factor (bFGF), and matrix metalloproteinases (MMPs) (Jiang et al., 2020; Ma et al., 2020). Recent studies have shown that the inhibition of angiogenesis by drug or antibodytargeting angiogenic growth factors has a potential therapeutic effect on many solid tumors (Pang et al., 2010; Yang M. et al., 2021). Clinical studies have shown that antiangiogenic therapy may have the greatest initial impact on tumor growth and metastasis (Heier et al., 2016; Scott et al., 2017). A number of studies have found that Chinese traditional medicines such as Qingdu granule, Oridonin (from Rabdosia rubescens), Ginsenoside Rd (from Panax ginseng C. A. Mey.), Arctigenin (from Arctium lappa L.), and cyperenoic acid (from Croton crassifolius Geiseler) can inhibit the tube formation of HUVECs in vitro and exhibit antiangiogenesis of breast cancer in vivo by inhibiting the expression of angiogenic growth factors and HIF-1a/VEGF signal pathway (Wang et al., 2017; Zhang et al., 2017; Li C. et al., 2018; Zhao et al., 2018). Tanshinone IIA is one of the major components from Chinese traditional medicine Danshen. Tanshinone IIA inhibits the migration and tube formation of bone marrow-derived endothelial progenitor cells (EPCs) in vitro and downregulates VEGF-mediated angiogenesis in the chick embryo chorioallantoic membrane (CAM) model (Lee et al., 2017). Studies showed that tanshinone IIA reduces the expression level of proangiogenic factors such as VEGF and COX2 to inhibit angiogenesis in human colorectal cancer (Zhou et al., 2012; Sui et al., 2017; Zhou L. et al., 2020). In ovarian cancer, tanshinone IIA significantly downregulates VEGF and cyclooxygenase-2 (COX2) mRNA expression in vitro and in vivo (Zhou et al., 2020c). Tanshinone IIA and tanshinone I suppress angiogenesis by targeting the protein kinase domains of VEGF/VEGFR2 and reducing the expression of VEGF in the human lung cancer cells (Tung et al., 2013; Xie et al., 2015).

In breast cancer, tanshinone I downregulates hypoxia-inducible factor 1alpha (HIF-1a) and VEGF in breast cancer MDA-MB-231 cells, and tanshinone IIA inhibits the angiogenesis and growth of 


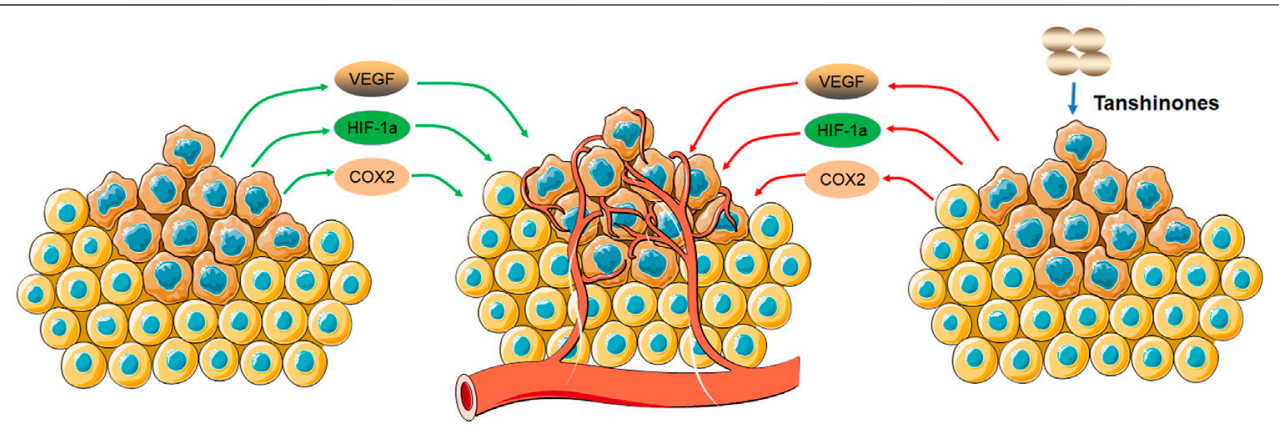

FIGURE 5 | Molecular mechanism of tanshinones inhibiting angiogenesis. Tanshinones inhibit protein expression of VEGF, COX2, and HIF-1a. VEGF, vascular endothelial growth factor; COX2, cytochrome C oxidase II; HIF-1a, hypoxia-inducible factor 1 subunit alpha.

human breast cancer xenografts in nude mice through suppression of HIF-1 $\alpha$ and VEGF (Nizamutdinova et al., 2008a; Liao et al., 2020). Cryptotanshinone is one of the tanshinones isolated from the roots of the plant Salvia miltiorrhiza Bunge (Danshen). It has been shown that it downregulates the levels of VEGF mRNA and protein in a dosedependent manner in U2OS osteosarcoma cells and resists angiogenesis ability by inhibiting the tube formation assay in vitro (Xu et al., 2016). Therefore, active components from Salvia miltiorrhiza Bunge (Danshen) had antiangiogenic effects by inhibiting mRNA and protein expression of proangiogenic growth factors such as VEGF and COX2. Our study showed that tanshinone IIA inhibits angiogenesis by reducing VEGF and COX2 mRNA expression of ovarian cancer cells in vitro and in vivo (Zhou et al., 2020c).

In summary, tanshinones can inhibit cell growth and tumor growth by regulating the expression of proangiogenic growth factors and hypoxia-inducible factor 1alpha (HIF-1a) (Figure 5).

\section{CONCLUSION AND EXPECTATION}

During the past few decades, many studies have demonstrated the potential benefits of tanshinones for the treatment of gynecological tumors in vitro and in vivo. Mechanism studies showed that tanshinones inhibit cancer cell proliferation and tumor growth by inducing cancer cell apoptosis and cell cycle arrest, inhibiting metastasis phenotype, enhancing chemosensitivity and radiosensitivity, and blocking the angiogenesis of tumor. In addition to the characteristics of malignant proliferation, strong invasion and metastasis, and drug resistance, metabolic reprogramming is another main feature of tumor cells and also the hotspot and focus of tumor research. Metabolic reprogramming of tumor cells refers to the raw materials needed by cells to change their metabolic phenotype in harsh environment, so as to quickly produce adenosine triphosphate (ATP), synthesize macromolecular substances, maintain the redox state of cells, and finally realize the proliferation of tumor cells. Metabolic reprogramming of tumor cells enhances the restriction on glucose and amino acids, obtains nutrients for cell survival through other ways, uses glycolysis and tricarboxylic acid cycle intermediates for biosynthesis, and changes key metabolic enzymes of cell metabolism. Although not all tumor tissues have these characteristics, studies have found that abnormal metabolic phenotypes such as glycolysis, fatty acid metabolism, and glutamine decomposition generally exist in tumor cells. Our study showed that tanshinone I can regulate metabolic reprogramming of tumor cells. However, the specific molecular mechanism is not completely clear. In the future, the antitumor molecular mechanism of tanshinones will be further expanded to provide a new basis for the clinical application of tanshinones.

In addition, cryptotanshinone, tanshinone I, dihydrotanshinone I, and tanshinone IIA have low aqueous solubility and poor membrane permeability, which affected drug absorption (Hao et al., 2006; Wang et al., 2007; Wu et al., 2018). Many studies have demonstrated that tanshinones have poor bioavailability by oral administration or the gavage method (Zhang et al., 2006). For example, the bioavailability of cryptotanshinone in rats was approximately $2.1 \%$ by oral administration and $10.6 \%$ by intraperitoneal administration (Zhang et al., 2006). Based on these limitations, it is necessary to optimize tanshinone's structure and increase antitumor potency and drug-like properties. A recent study identified two tanshinone analogs, which increased antitumor potency as compared to the natural tanshinones both Tan-I and Tan-IIA (Gain et al., 2021).

In summary, tanshinones have shown many antitumor mechanisms and have higher potential in the treatment of female breast cancer and gynecological cancer.

\section{AUTHOR CONTRIBUTIONS}

$\mathrm{PC}, \mathrm{YC}$, and ZJ contributed to conception, figure, and revision. $\mathrm{PC}, \mathrm{YC}$, and $\mathrm{ZJ}$ contributed to writing and revising the draft.

\section{FUNDING}

This study was funded by National Natural Science Foundation of China (No. 81891012, U19A2010) and the Experimental Formulary Sichuan Youth Science and Technology Innovation Research Team (2020JDTD0022). 


\section{REFERENCES}

Amant, F., Berveiller, P., Boere, I. A., Cardonick, E., Fruscio, R., Fumagalli, M., et al. (2019). Gynecologic Cancers in Pregnancy: Guidelines Based on a Third International Consensus Meeting. Ann. Oncol. 30, 1601-1612. doi:10.1093/ annonc/mdz228

Burstein, H. J. (2020). Systemic Therapy for Estrogen Receptor-Positive, HER2Negative Breast Cancer. N. Engl. J. Med. 383, 2557-2570. doi:10.1056/ NEJMra1307118

Chang, C. C., Kuan, C. P., Lin, J. Y., Lai, J. S., and Ho, T. F. (2015). Tanshinone IIA Facilitates TRAIL Sensitization by Up-Regulating DR5 through the ROS-JNKCHOP Signaling Axis in Human Ovarian Carcinoma Cell Lines. Chem. Res. Toxicol. 28, 1574-1583. doi:10.1021/acs.chemrestox.5b00150

Coughlin, S. S. (2019). Epidemiology of Breast Cancer in Women. Adv. Exp. Med. Biol. 1152, 9-29. doi:10.1007/978-3-030-20301-6_2

DeSantis, C., Ma, J., Bryan, L., and Jemal, A. (2014). Breast Cancer Statistics, 2013. CA Cancer J. Clin. 64, 52-62. doi:10.3322/caac.21203

Dun, S., and Gao, L. (2019). Tanshinone I Attenuates Proliferation and Chemoresistance of Cervical Cancer in a KRAS-Dependent Manner. J. Biochem. Mol. Toxicol. 33, e22267. doi:10.1002/jbt.22267

Folkman, J. (2002). Role of Angiogenesis in Tumor Growth and Metastasis. Semin. Oncol. 29, 15-18. doi:10.1053/sonc.2002.37263

Fu, P., Du, F., Chen, W., Yao, M., Lv, K., and Liu, Y. (2014). Tanshinone IIA Blocks Epithelial-Mesenchymal Transition through HIF-1a Downregulation, Reversing Hypoxia-Induced Chemotherapy Resistance in Breast Cancer Cell Lines. Oncol. Rep. 31, 2561-2568. doi:10.3892/or.2014.3140

Gain, C., Sarkar, A., Bural, S., Rakshit, M., Banerjee, J., Dey, A., et al. (2021). Identification of Two Novel Thiophene Analogues as Inducers of Autophagy Mediated Cell Death in Breast Cancer Cells. Bioorg. Med. Chem. 37, 116112. doi:10.1016/j.bmc.2021.116112

Gong, Y., Li, Y., Abdolmaleky, H. M., Li, L., and Zhou, J. R. (2012). Tanshinones Inhibit the Growth of Breast Cancer Cells through Epigenetic Modification of Aurora A Expression and Function. PLoS One 7, e33656. doi:10.1371/ journal.pone. 0033656

Gu, G., Dustin, D., and Fuqua, S. A. (2016). Targeted Therapy for Breast Cancer and Molecular Mechanisms of Resistance to Treatment. Curr. Opin. Pharmacol. 31, 97-103. doi:10.1016/j.coph.2016.11.005

Hao, H., Wang, G., Cui, N., Li, J., Xie, L., and Ding, Z. (2006). Pharmacokinetics, Absorption and Tissue Distribution of Tanshinone IIA Solid Dispersion. Planta Med. 72, 1311-1317. doi:10.1055/s-2006-951698

Heier, J. S., Bressler, N. M., Avery, R. L., Bakri, S. J., Boyer, D. S., Brown, D. M., et al. (2016). Comparison of Aflibercept, Bevacizumab, and Ranibizumab for Treatment of Diabetic Macular Edema: Extrapolation of Data to Clinical Practice. JAMA Ophthalmol. 134, 95-99. doi:10.1001/ jamaophthalmol.2015.4110

Jiang, G., Liu, J., Ren, B., Zhang, L., Owusu, L., Liu, L., et al. (2017). Anti-Tumor and Chemosensitization Effects of Cryptotanshinone Extracted from Salvia Miltiorrhiza Bge. On Ovarian Cancer Cells In Vitro. J. Ethnopharmacol 205, 33-40. doi:10.1016/j.jep.2017.04.026

Jiang, X., Wang, J., Deng, X., Xiong, F., Zhang, S., Gong, Z., et al. (2020). The Role of Microenvironment in Tumor Angiogenesis. J. Exp. Clin. Cancer Res. 39, 204. doi:10.1186/s13046-020-01709-5

Jiao, J. W., and Wen, F. (2011). Tanshinone IIA Acts via P38 MAPK to Induce Apoptosis and the Down-Regulation of ERCC1 and Lung-Resistance Protein in Cisplatin-Resistant Ovarian Cancer Cells. Oncol. Rep. 25, 781-788. doi:10.3892/ or.2010.1107

Jing, J., Zheng, H., Wang, J., Lin, P., Zhang, J., Xiong, Z. J., et al. (2007). Growth Inhibition and Multidrug Resistance-Reversing Effect of Tanshinone I A on Human Breast Cancer Cell with Estrogen Receptor Negative. Sichuan Da Xue Xue Bao Yi Xue Ban 38, 391-395.

Kim, H., Xu, H., George, E., Hallberg, D., Kumar, S., Jagannathan, V., et al. (2020). Combining PARP with ATR Inhibition Overcomes PARP Inhibitor and Platinum Resistance in Ovarian Cancer Models. Nat. Commun. 11, 3726. doi:10.1038/s41467-020-17127-2

Lee, H. P., Liu, Y. C., Chen, P. C., Tai, H. C., Li, T. M., Fong, Y. C., et al. (2017). Tanshinone IIA Inhibits Angiogenesis in Human Endothelial Progenitor Cells
In Vitro and In Vivo. Oncotarget 8, 109217-109227. doi:10.18632/ oncotarget.22649

Li, K., and Lai, H. (2017). TanshinoneIIA Enhances the Chemosensitivity of Breast Cancer Cells to Doxorubicin through Down-Regulating the Expression of MDR-Related ABC Transporters. Biomed. Pharmacother. 96, 371-377. doi:10.1016/j.biopha.2017.10.016

Li, C., Wang, Q., Shen, S., Wei, X., and Li, G. (2018a). Oridonin Inhibits VEGF-AAssociated Angiogenesis and Epithelial-Mesenchymal Transition of Breast Cancer In Vitro and In Vivo. Oncol. Lett. 16, 2289-2298. doi:10.3892/ ol.2018.8943

Li, N., Yang, L., Zhang, B., and Chen, S. (2018b). Tanshinone IIA Effects on Ovarian Cancer Cell Line. J. Pharm. Pharmacol. 70, 1369-1377. doi:10.1111/ jphp.12961

Li, Q., Zhang, J., Liang, Y., Mu, W., Hou, X., Ma, X., et al. (2018c). Tanshinone L Exhibits Anticancer Effects in Human Endometrial Carcinoma HEC-1-A Cells via Mitochondrial Mediated Apoptosis, Cell Cycle Arrest and Inhibition of JAK/STAT Signalling Pathway. J. BUON 23, 1092-1096.

Li, J., Rong, M. H., Dang, Y. W., He, R. Q., Lin, P., Yang, H., et al. (2019a). Differentially Expressed Gene Profile and Relevant Pathways of the Traditional Chinese Medicine Cinobufotalin on MCF-7 Breast Cancer Cells. Mol. Med. Rep. 19, 4256-4270. doi:10.3892/mmr.2019.10062

Li, K., Liu, W., Zhao, Q., Wu, C., Fan, C., Lai, H., et al. (2019b). Combination of Tanshinone IIA and Doxorubicin Possesses Synergism and Attenuation Effects on Doxorubicin in the Treatment of Breast Cancer. Phytother Res. 33, 1658-1669. doi:10.1002/ptr.6353

Li, Q., Qin, T., Bi, Z., Hong, H., Ding, L., Chen, J., et al. (2020). Rac1 Activates NonOxidative Pentose Phosphate Pathway to Induce Chemoresistance of Breast Cancer. Nat. Commun. 11, 1456. doi:10.1038/s41467-020-15308-7

Li, S., Wu, C., Fan, C., Zhang, P., Yu, G., and Li, K. (2021). Tanshinone II A Improves the Chemosensitivity of Breast Cancer Cells to Doxorubicin by Inhibiting Beta-Catenin Nuclear Translocation. J. Biochem. Mol. Toxicol. 35, e22620. doi:10.1002/jbt.22620

Liao, X., Gao, Y., Liu, J., Tao, L., Xie, J., Gu, Y., et al. (2020). Combination of Tanshinone IIA and Cisplatin Inhibits Esophageal Cancer by Downregulating NFKb/cox-2/VEGF Pathway. Front. Oncol. 10, 1756. doi:10.3389/fonc.2020.01756

Lin, H., Zheng, L., Li, S., Xie, B., Cui, B., Xia, A., et al. (2018). Cytotoxicity of Tanshinone IIA Combined with Taxol on Drug-Resist Breast Cancer Cells MCF-7 through Inhibition of Tau. Phytother Res. 32, 667-671. doi:10.1002/ ptr.6014

Liu, Y. T., Hsiao, C. H., Tzang, B. S., and Hsu, T. C. (2019a). In Vitro and In Vivo Effects of Traditional Chinese Medicine Formula T33 in Human Breast Cancer Cells. BMC Complement. Altern. Med. 19, 211. doi:10.1186/s12906-019-2630-5

Liu, Z., Zhu, W., Kong, X., Chen, X., Sun, X., Zhang, W., et al. (2019b). Tanshinone IIA Inhibits Glucose Metabolism Leading to Apoptosis in Cervical Cancer. Oncol. Rep. 42, 1893-1903. doi:10.3892/or.2019.7294

Liu, J., Jiao, X., and Gao, Q. (2020). Neoadjuvant Chemotherapy-Related Platinum Resistance in Ovarian Cancer. Drug Discov. Today 25, 1232-1238. doi:10.1016/ j.drudis.2020.04.015

Lu, Q., Zhang, P., Zhang, X., and Chen, J. (2009). Experimental Study of the AntiCancer Mechanism of Tanshinone IIA against Human Breast Cancer. Int. J. Mol. Med. 24, 773-780. doi:10.3892/ijmm_00000291

Luo, J., Meng, X., Su, J., Ma, H., Wang, W., Fang, L., et al. (2018). Biotin-Modified Polylactic- Co-Glycolic Acid Nanoparticles with Improved Antiproliferative Activity of 15,16-Dihydrotanshinone I in Human Cervical Cancer Cells. J. Agric. Food Chem. 66, 9219-9230. doi:10.1021/acs.jafc.8b02698

Ma, Q., Reiter, R. J., and Chen, Y. (2020). Role of Melatonin in Controlling Angiogenesis under Physiological and Pathological Conditions. Angiogenesis 23, 91-104. doi:10.1007/s10456-019-09689-7

Munagala, R., Aqil, F., Jeyabalan, J., and Gupta, R. C. (2015). Tanshinone IIA Inhibits Viral Oncogene Expression Leading to Apoptosis and Inhibition of Cervical Cancer. Cancer Lett. 356, 536-546. doi:10.1016/ j.canlet.2014.09.037

Nicolin, V., Fancellu, G., and Valentini, R. (2014). Effect of Tanshinone II on Cell Growth of Breast Cancer Cell Line Type MCF-7 and MD-MB-231. Ital. J. Anat. Embryol. 119, 38-43.

Nizamutdinova, I. T., Lee, G. W., Lee, J. S., Cho, M. K., Son, K. H., Jeon, S. J., et al. (2008a). Tanshinone I Suppresses Growth and Invasion of Human Breast 
Cancer Cells, MDA-MB-231, through Regulation of Adhesion Molecules. Carcinogenesis 29, 1885-1892. doi:10.1093/carcin/bgn151

Nizamutdinova, I. T., Lee, G. W., Son, K. H., Jeon, S. J., Kang, S. S., Kim, Y. S., et al. (2008b). Tanshinone I Effectively Induces Apoptosis in Estrogen ReceptorPositive (MCF-7) and Estrogen Receptor-Negative (MDA-MB-231) Breast Cancer Cells. Int. J. Oncol. 33, 485-491. doi:10.3892/ijo_00000031

Pan, T. L., Hung, Y. C., Wang, P. W., Chen, S. T., Hsu, T. K., Sintupisut, N., et al. (2010). Functional Proteomic and Structural Insights into Molecular Targets Related to the Growth Inhibitory Effect of Tanshinone IIA on HeLa Cells. Proteomics 10, 914-929. doi:10.1002/pmic.200900178

Pan, T. L., Wang, P. W., Hung, Y. C., Huang, C. H., and Rau, K. M. (2013). Proteomic Analysis Reveals Tanshinone IIA Enhances Apoptosis of Advanced Cervix Carcinoma CaSki Cells through Mitochondria Intrinsic and Endoplasmic Reticulum Stress Pathways. Proteomics 13, 3411-3423. doi:10.1002/pmic.201300274

Pang, X., Yi, Z., Zhang, J., Lu, B., Sung, B., Qu, W., et al. (2010). Celastrol Suppresses Angiogenesis-Mediated Tumor Growth through Inhibition of AKT/ Mammalian Target of Rapamycin Pathway. Cancer Res. 70, 1951-1959. doi:10.1158/0008-5472.CAN-09-3201

Pondé, N. F., Zardavas, D., and Piccart, M. (2019). Progress in Adjuvant Systemic Therapy for Breast Cancer. Nat. Rev. Clin. Oncol. 16, 27-44. doi:10.1038/ s41571-018-0089-9

Qin, J., Shi, H., Xu, Y., Zhao, F., and Wang, Q. (2018). Tanshinone IIA Inhibits Cervix Carcinoma Stem Cells Migration and Invasion via Inhibiting YAP Transcriptional Activity. Biomed. Pharmacother. 105, 758-765. doi:10.1016/ j.biopha.2018.06.028

Scott, I. U., VanVeldhuisen, P. C., Ip, M. S., Blodi, B. A., Oden, N. L., Awh, C. C., et al. (2017). Effect of Bevacizumab vs Aflibercept on Visual Acuity Among Patients with Macular Edema Due to Central Retinal Vein Occlusion: The SCORE2 Randomized Clinical Trial. JAMA 317, 2072-2087. doi:10.1001/ jama.2017.4568

Su, C. C., and Lin, Y. H. (2008). Tanshinone IIA Inhibits Human Breast Cancer Cells through Increased Bax to Bcl-xL Ratios. Int. J. Mol. Med. 22, 357-361. doi:10.3892/ijmm_00000030

Su, C. C., Chien, S. Y., Kuo, S. J., Chen, Y. L., Cheng, C. Y., and Chen, D. R. (2012). Tanshinone IIA Inhibits Human Breast Cancer MDA-MB-231 Cells by Decreasing LC3-II, Erb-B2 and NF-kBp65. Mol. Med. Rep. 5, 1019-1022. doi:10.3892/mmr.2012.756

Sui, H., Zhao, J., Zhou, L., Wen, H., Deng, W., Li, C., et al. (2017). Tanshinone IIA Inhibits $\beta$-Catenin/VEGF-Mediated Angiogenesis by Targeting TGF-B1 in Normoxic and HIF-1 $\alpha$ in Hypoxic Microenvironments in Human Colorectal Cancer. Cancer Lett. 403, 86-97. doi:10.1016/j.canlet.2017.05.013

Tong, W., Guo, J., and Yang, C. (2020). Tanshinone II A Enhances Pyroptosis and Represses Cell Proliferation of HeLa Cells by Regulating miR-145/GSDMD Signaling Pathway. Biosci. Rep. 40, BSR20200259. doi:10.1042/BSR20200259

Tsuyoshi, H., Inoue, D., Kurokawa, T., and Yoshida, Y. (2020). Hyperthermic Intraperitoneal Chemotherapy (HIPEC) for Gynecological Cancer. J. Obstet. Gynaecol. Res. 46, 1661-1671. doi:10.1111/jog.14391

Tung, Y. T., Chen, H. L., Lee, C. Y., Chou, Y. C., Lee, P. Y., Tsai, H. C., et al. (2013). Active Component of Danshen (Salvia Miltiorrhiza Bunge), Tanshinone I, Attenuates Lung Tumorigenesis via Inhibitions of VEGF, Cyclin A, and Cyclin B Expressions. Evid. Based Complement. Alternat Med. 2013, 319247. doi:10.1155/2013/319247

Wang, X., Wei, Y., Yuan, S., Liu, G., Lu, Y., Zhang, J., et al. (2005). Potential Anticancer Activity of Tanshinone IIA against Human Breast Cancer. Int. J. Cancer 116, 799-807. doi:10.1002/ijc.20880

Wang, J., Wang, X., Jiang, S., Yuan, S., Lin, P., Zhang, J., et al. (2007). Growth Inhibition and Induction of Apoptosis and Differentiation of Tanshinone IIA in Human Glioma Cells. J. Neurooncol. 82, 11-21. doi:10.1007/s11060-006-9242-x

Wang, P., Solorzano, W., Diaz, T., Magyar, C. E., Henning, S. M., and Vadgama, J. V. (2017). Arctigenin Inhibits Prostate Tumor Cell Growth In Vitro and In Vivo. Clin. Nutr. Exp. 13, 1-11. doi:10.1016/j.yclnex.2017.04.001

Wang, X., Fan, J., Ding, X., Sun, Y., Cui, Z., and Liu, W. (2019). Tanshinone I Inhibits IL-1 $\beta$-Induced Apoptosis, Inflammation and Extracellular Matrix Degradation in Chondrocytes CHON-001 Cells and Attenuates Murine Osteoarthritis. Drug Des. Devel Ther. 13, 3559-3568. doi:10.2147/ DDDT.S216596

Wang, X., Xu, X., Jiang, G., Zhang, C., Liu, L., Kang, J., et al. (2020). Dihydrotanshinone I Inhibits Ovarian Cancer Cell Proliferation and
Migration by Transcriptional Repression of PIK3CA Gene. J. Cel Mol Med 24, 11177-11187. doi:10.1111/jcmm.15660

Wu, Q., Zheng, K., Huang, X., Li, L., and Mei, W. (2018). Tanshinone-IIA-Based Analogues of Imidazole Alkaloid Act as Potent Inhibitors to Block Breast Cancer Invasion and Metastasis In Vivo. J. Med. Chem. 61, 10488-10501. doi:10.1021/acs.jmedchem.8b01018

Xie, J., Liu, J., Liu, H., Liang, S., Lin, M., Gu, Y., et al. (2015). The Antitumor Effect of Tanshinone IIA on Anti-Proliferation and Decreasing VEGF/VEGFR2 Expression on the Human Non-small Cell Lung Cancer A549 Cell Line. Acta Pharm. Sin B 5, 554-563. doi:10.1016/j.apsb.2015.07.008

Xu, Y., Ji, Q., Zhang, Q., and Wang, Y. (2016). Cryptotanshinone Down-Regulates the Expression of VEGF and Inhibits Angiogenesis in U2OS Osteosarcoma Cells. Xi Bao Yu Fen Zi Mian Yi Xue Za Zhi 32, 29-33. doi:10.13423/ j.cnki.cjcmi.007616

Yadav, B. S., Sharma, S. C., Patel, F. D., Rai, B., and Ghoshal, S. (2017). Gynecological Cancer as a Second Malignancy in Patients with Breast Cancer. Int. J. Gynecol. Cancer 27, 1298-1304. doi:10.1097/IGC.0000000000000993

Yan, M. Y., Chien, S. Y., Kuo, S. J., Chen, D. R., and Su, C. C. (2012). Tanshinone IIA Inhibits BT-20 Human Breast Cancer Cell Proliferation through Increasing Caspase 12, GADD153 and Phospho-P38 Protein Expression. Int. J. Mol. Med. 29, 855-863. doi:10.3892/ijmm.2012.908

Yang, Y., Cao, Y., Chen, L., Liu, F., Qi, Z., Cheng, X., et al. (2018). Cryptotanshinone Suppresses Cell Proliferation and Glucose Metabolism via STAT3/SIRT3 Signaling Pathway in Ovarian Cancer Cells. Cancer Med. 7, 4610-4618. doi:10.1002/cam4.1691

Yang, M., Su, Y., Wang, Z., Du, D., Wei, S., Liao, Z., et al. (2021a). C118P, a Novel Microtubule Inhibitor with Anti-angiogenic and Vascular Disrupting Activities, Exerts Anti-tumor Effects against Hepatocellular Carcinoma. Biochem. Pharmacol. 190, 114641. doi:10.1016/j.bcp.2021.114641

Yang, Z., Zhang, Q., Yu, L., Zhu, J., Cao, Y., and Gao, X. (2021b). The Signaling Pathways and Targets of Traditional Chinese Medicine and Natural Medicine in Triple-Negative Breast Cancer. J. Ethnopharmacol 264, 113249. doi:10.1016/ j.jep.2020.113249

Ye, Y., Xu, W., and Zhong, W. (2010). Effects of Cryptotanshinone on Proliferation and Apoptosis of Hela Cell Line of Cervical Cancer. Zhongguo Zhong Yao Za Zhi 35, 118-121. doi:10.4268/cjcmm20100125

Yousefi, H., Vatanmakanian, M., Mahdiannasser, M., Mashouri, L., Alahari, N. V., Monjezi, M. R., et al. (2021). Understanding the Role of Integrins in Breast Cancer Invasion, Metastasis, Angiogenesis, and Drug Resistance. Oncogene 40, 1043-1063. doi:10.1038/s41388-020-01588-2

Zaker, A., Asili, J., Abrishamchi, P., Tayarani-Najaran, Z., and Mousavi, S. H. (2017). Cytotoxic and Apoptotic Effects of Root Extract and Tanshinones Isolated from Perovskiaabrotanoides Kar. Iran J. Basic Med. Sci. 20, 1377-1384. doi:10.22038/IJBMS.2017.9568

Zhang, J., Huang, M., Guan, S., Bi, H. C., Pan, Y., Duan, W., et al. (2006). A Mechanistic Study of the Intestinal Absorption of Cryptotanshinone, the Major Active Constituent of Salvia Miltiorrhiza. J. Pharmacol. Exp. Ther. 317, 1285-1294. doi:10.1124/jpet.105.100701

Zhang, E., Shi, H., Yang, L., Wu, X., and Wang, Z. (2017). Ginsenoside Rd Regulates the Akt/mTOR/p70S6K Signaling cascade and Suppresses Angiogenesis and Breast Tumor Growth. Oncol. Rep. 38, 359-367. doi:10.3892/or.2017.5652

Zhang, X., Zhou, Y., and Gu, Y. E. (2019). Tanshinone IIA Induces Apoptosis of Ovarian Cancer Cells In Vitro and In Vivo through Attenuation of PI3K/AKT/ JNK Signaling Pathways. Oncol. Lett. 17, 1896-1902. doi:10.3892/ol.2018.9744

Zhao, X., Liu, J., Feng, L., Ge, S., Yang, S., Chen, C., et al. (2018). Anti-Angiogenic Effects of Qingdu Granule on Breast Cancer through Inhibiting NFAT Signaling Pathway. J. Ethnopharmacol 222, 261-269. doi:10.1016/j.jep.2018.01.013

Zhou, L. H., Hu, Q., Sui, H., Ci, S. J., Wang, Y., Liu, X., et al. (2012). Tanshinone IIAa Inhibits Angiogenesis through Down Regulation of COX-2 in Human Colorectal Cancer. Asian Pac. J. Cancer Prev. 13, 4453-4458. doi:10.7314/ apjcp.2012.13.9.4453

Zhou, J., Jiang, Y. Y., Chen, H., Wu, Y. C., and Zhang, L. (2020a). Tanshinone I Attenuates the Malignant Biological Properties of Ovarian Cancer by Inducing Apoptosis and Autophagy via the Inactivation of PI3K/AKT/mTOR Pathway. Cell Prolif 53, e12739. doi:10.1111/cpr.12739

Zhou, J., Jiang, Y. Y., Wang, H. P., Chen, H., Wu, Y. C., Wang, L., et al. (2020b). Natural Compound Tan-I Enhances the Efficacy of Paclitaxel Chemotherapy in Ovarian Cancer. Ann. Transl Med. 8, 752. doi:10.21037/atm-20-4072 
Zhou, J., Jiang, Y. Y., Wang, X. X., Wang, H. P., Chen, H., Wu, Y. C., et al. (2020c). Tanshinone IIA Suppresses Ovarian Cancer Growth through Inhibiting Malignant Properties and Angiogenesis. Ann. Transl Med. 8, 1295. doi:10.21037/atm-20-5741

Zhou, L., Sui, H., Wang, T., Jia, R., Zhang, Z., Fu, J., et al. (2020d). Tanshinone IIA Reduces Secretion of Pro-Angiogenic Factors and Inhibits Angiogenesis in Human Colorectal Cancer. Oncol. Rep. 43, 1159-1168. doi:10.3892/ or.2020.7498

Conflict of Interest: The authors declare that the research was conducted in the absence of any commercial or financial relationships that could be construed as a potential conflict of interest.
Publisher's Note: All claims expressed in this article are solely those of the authors and do not necessarily represent those of their affiliated organizations, or those of the publisher, the editors, and the reviewers. Any product that may be evaluated in this article, or claim that may be made by its manufacturer, is not guaranteed or endorsed by the publisher.

Copyright $\odot 2022$ Jin, Chenghao and Cheng. This is an open-access article distributed under the terms of the Creative Commons Attribution License (CC BY). The use, distribution or reproduction in other forums is permitted, provided the original author(s) and the copyright owner(s) are credited and that the original publication in this journal is cited, in accordance with accepted academic practice. No use, distribution or reproduction is permitted which does not comply with these terms. 